\title{
Variaciones de inervación del nervio peroneo superficial y sural en la zona proximal del pie. Estudio anatómico cadavérico
}

\author{
Variations of innervation of superficial peroneal nerve and sural nerve in distal leg and foot. \\ An anatomic cadaveric study
}

\author{
Francisco Casado Hernández 1 , Ángel Manuel Orejana García² y Javier Pascual Huerta ${ }^{3}$ \\ ${ }^{1}$ Clínica Principium, Soria. ${ }^{2}$ Universidad Complutense de Madrid, Madrid. ${ }^{3}$ Clínica del Pie Elcano, Bilbao
}

Palabras clave:

Nervio peroneo superficial, nervio sural, nervio cutáneo dorsal lateral, nervio cutáneo dorsal intermedio, nervio cutáneo dorsal medial, variaciones anatómicas, inervación del pie, estudio cadavérico.

\section{Resumen}

Introducción: Existen variaciones importantes en cuanto a la distribución y territorio de inervación del nervio peroneo superficial (NPS) y nervio sural (NS) en la cara dorsolateral del pie y la pierna, habiendo sido descritos diferentes patrones de inervación. La literatura no es concluyente en cuanto a los porcentajes de presentación de estos patrones.

Material y métodos: Se realizó un estudio cadavérico sobre 49 piezas anatómicas con un diseño descriptivo transversal. El estudio se realizó sobre piezas recientemente descongeladas que se diseccionaron de forma sistemática. Se estimó la prevalencia de variaciones anatómicas con respecto a tres patrones de distribución y se tomaron mediciones de las perforaciones del NPS y del NS respecto al maléolo peroneo.

Resultados: La distribución encontrada fue: un 48.89 \% (IC 95 \% de $33.70 \%$ a 64.08 \%); presentaba una distribución tipo I (el NPS perfora la fascia profunda y posteriormente se divide en nervio cutáneo dorsal intermedio [NCDI] y nervio cutáneo dorsal medial [NCDM]), un $33.33 \%$ (IC $95 \%$ de $19.01 \%$ a $47.66 \%$ ) presentaba una distribución tipo II (el NPS se divide en el compartimento profundo y el NCDI y NCDM perforan la fascia como estructuras independientes) y un $17,78 \%$ (IC $95 \%$ de $6,16 \%$ a $29,39 \%$ ) presentaba una distribución tipo III (el NPS perfora la fascia profunda y sigue el recorrido del NCDM y el NCDI está ausente).

Conclusión: El presente estudio aporta datos de patrones de inervación dorsal del pie en una muestra de 49 piezas cadavéricas. Esta información puede resultar de utilidad en los abordajes quirúrgicos y anestésicos del pie.

Keywords:

Superficial peroneal nerve, sural nerve, lateral dorsal cutaneous nerve, intermediate dorsal cutaneous nerve, medial dorsal cutaneous nerve, anatomic variations, foot innervation, cadaveric study.

\begin{abstract}
Introduction: There are important variations in the innervation of the dorsum of the foot and leg regarding the distribution of the superficial peroneal nerve (SPN) and sural nerve (SN). Although several patterns have been described, literature has not reach a consensus on the percentages of those patterns.

Materials and methods: A cadaveric anatomical study was performed on 49 fresh frozen lower limbs. The study had a transversal descriptive design. A systematic dissection was carried out on dorsum of the foot of the specimens and the estimated prevalence of anatomic variations taking as reference 3 patterns of distribution was calculated. Measurements of perforation of the SPN and SN were also taken.

Results: The patterns of distribution of SPN were: $48.89 \%$ (CI $95 \%$ from $33.70 \%$ to $64.08 \%$ ) had a type I distribution, where SPN pierces deep fascia and then it divides into medial dorsal cutaneous nerve (MDCN) and intermediate dorsal cutaneous nerve (IDCN); $33.33 \%$ (CI $95 \%$ from $19.01 \%$ to $47.66 \%$ ) had a type II distribution, where SPN bifurcates and then pierces the fascial layer separately as MDCN and IDCN; 17.78 \% (CI $95 \%$ from $6.16 \%$ to $29.39 \%$ ) had a type III distribution, where SPN pierces deep fascia, courses identical as MDCN and IDCN is absent.

Conclusion: The present study gives data on the pattern of distribution of the innervation of the dorsum of the foot and leg and its percentages in a sample of 49 feet. The information reported has important implications in surgical and anesthetic approaches of the foot and ankle.
\end{abstract}




\section{INTRODUCCIÓN}

El nervio peroneo superficial y el nervio sural son dos de los principales nervios que aportan inervación dorsal al pie. La descripción clásica del trayecto y territorio de inervación de estos nervios describe al nervio peroneo superficial atravesando la fascia profunda (fascia cruris) y haciéndose superficial en la cara anterolateral del tercio distal de la pierna para, posteriormente, dividirse en una rama medial y otra lateral. La rama medial es el nervio cutáneo dorsal medial que vuelve a dividirse, dando una rama medial para la cara medial del primer dedo y una rama lateral para el segundo espacio intermetatarsal. La rama lateral del nervio peroneo superficial es el nervio cutáneo dorsal intermedio, que da inervación al tercer y cuarto espacios intermetatarsales ${ }^{1-3}$ (Figura 1). Por su parte, el nervio sural se hace superficial en la zona posterior de la pierna y desciende distalmente en dirección levemente lateral para cruzar el tobillo entre el tendón de Aquiles y el maléolo lateral. Durante su recorrido da múltiples ramas que inervan la cara lateral y posterior del pie y la pierna y continúa distalmente su rama terminal hasta inervar la cara lateral del quinto dedo ${ }^{2,3}$ (Figura 1). No obstante, existe una gran variabilidad en el patrón de inervación dorsal del pie de unos individuos a otros y esta descripción clásica descrita y mostrada en la Figura 1 está únicamente presente en apenas la mitad de los individuos ${ }^{4-6}$, aspecto que puede comprometer en ocasiones los abordajes quirúrgicos y las técnicas anestésicas distales del pie y tobillo.
El trabajo clásico realizado por la Anatomical Society of Great Britain and Ireland, basado en la disección de 229 pies y publicado en $1891^{4}$, describió un total de 12 patrones diferentes de inervación para el dorso del pie, que se muestran en la Figura 2, aportando información estadística de su frecuencia. Este trabajo, que se mantiene hoy en día como una referencia importante para describir la variabilidad de la inervación dorsal del pie, mostró que, en términos generales, cuando el territorio inervado por el nervio sural aumenta, disminuye el del nervio cutáneo dorsal intermedio y viceversa. Aproximadamente un $25 \%$ de los especímenes examinados en dicho estudio mostraron ausencia del nervio cutáneo dorsal intermedio, siendo el nervio sural el nervio que suplía el territorio del nervio cutáneo dorsal intermedio en el dorso del pie. Asimismo, en los últimos 25 años diversos estudios ${ }^{7-13}$ han mostrado una gran variabilidad en cuanto a la frecuencia de los patrones de inervación de la zona dorsal del pie. Esto es especialmente llamativo en cuanto al porcentaje de presencia y/o ausencia del nervio cutáneo dorsal intermedio y al reemplazo de su territorio de inervación por el nervio sural. Una reciente revisión sistemática y metanálisis sobre los patrones de bifurcación y perforación de la fascia profunda del nervio peroneo superficial ( 665 miembros inferiores) ${ }^{13}$ mostró que la ausencia de nervio cutáneo dorsal intermedio ocurría únicamente en un 1,8\% de especímenes, por lo que el trabajo de la Anatomical Society of Great Britain and Ireland podría estar sobrevalorando este parámetro (que lo tasa en un $25 \%$ ). Es, por tanto, muy evidente la necesidad de realizar más estu-

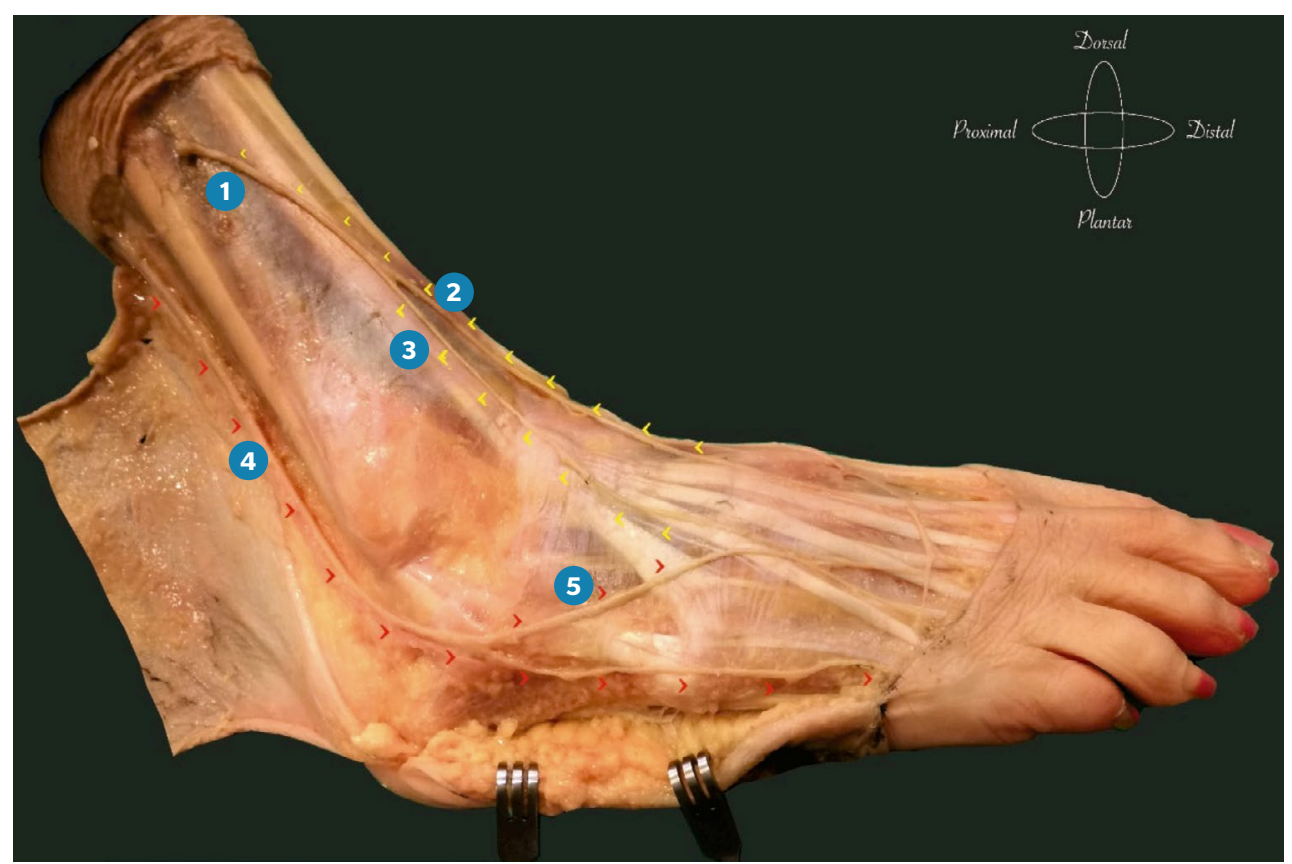

Figura 1. Anatomía del nervio peroneo superficial y sural en la cara anterolateral de la pierna y pie. Tipo I de la clasificación de Tomaszewski y cols. ${ }^{13}$.

1. Nervio peroneo superficial al atravesar la fascia cruris en la cara anterolateral de la pierna;

2. Nervio cutáneo dorsal medial; 3. Nervio cutáneo dorsal intermedio; 4. Nervio sural. 5. Rama anastomótica del nervio sural con el nervio cutáneo dorsal intermedio. 
90 REPORT OF COMMTTTEE OF COLLECTIVE INVESTIGATION OF
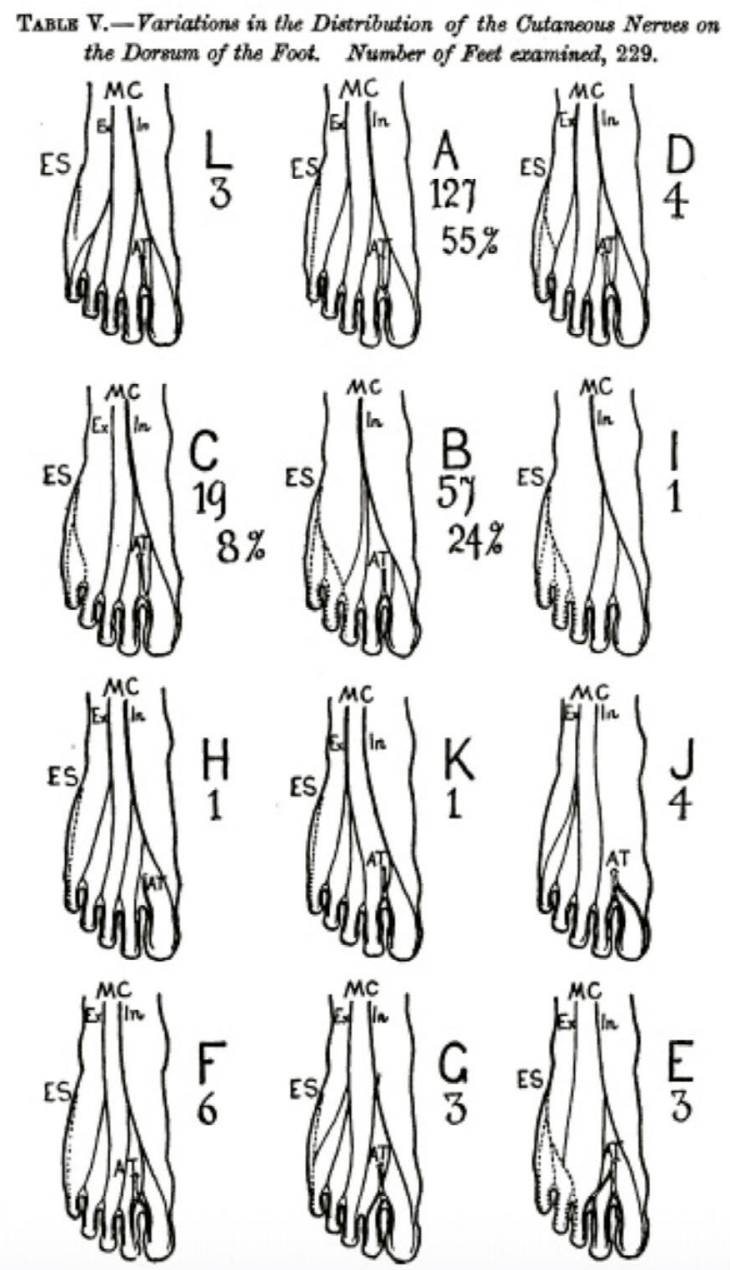

Figura 2. Tabla original obtenida de Anatomical Society of Great Britain and Ireland en: Thompson A. Second Annual Report of the Committee of Collective Investigation on the Distribution of Cutaneous Nerves on the Dorsum of the Foot.) Anat Physiol. 1891;26(Part 1):89-101. Tabla V.

dios anatómicos que describan las variaciones anatómicas de estos nervios y sus frecuencias.

El objetivo principal del presente trabajo consiste en valorar el patrón de inervación de la zona dorsolateral del pie en una muestra de especímenes cadavéricos, prestando especial interés en la presencia o ausencia del nervio cutáneo dorsal intermedio y a su suplantación o reemplazo de su territorio de inervación por el nervio sural. El objetivo secundario consiste en realizar una medición tomando como referencia el maléolo peroneo de la localización del punto de perforación de la fascia profunda del nervio peroneo superficial y/o de sus ramas terminales (nervio cutáneo dorsal medial e intermedio) y también del nervio sural con respecto al maléolo peroneo. Estas mediciones podrían ayudar a la hora de plantear puntos de referencia para realizar bloqueos anestésicos en el pie o para planificar las localizaciones de incisiones más seguras que no comprometan las estructuras nerviosas.

\section{MATERIAL Y MÉTODOS}

El presente trabajo es un estudio cadavérico con un diseño puramente descriptivo transversal. Para la redacción del presente artículo se siguió el listado AQUA (Anatomical QUality Assurance checklist) recomendado en la red EQUATOR como guía para el reporte de estudios anatómicos originales ${ }^{14}$.

El estudio se realizó sobre piezas cadavéricas recientemente descongeladas que se diseccionaron de forma sistemática. Las piezas cadavéricas del estudio estaban siendo utilizadas para la realización de un seminario de disección anatómica para podólogos que se llevó a cabo en una sala de disección a lo largo de un fin de semana completo, y que englobaba toda la disección anatómica del pie y tobillo. Las mediciones utilizadas en el estudio se tomaron de las piezas cadavéricas utilizadas en cuatro de estos seminarios, que fueron realizados en las siguientes lugares y fechas: Valencia (febrero de 2017 y enero de 2018), Pamplona (noviembre de 2018) y Oviedo (diciembre de 2018). Se utilizaron todas las piezas anatómicas con las que se realizó el seminario. Ninguna de las piezas anatómicas del estudio mostraba evidencias de intervenciones quirúrgicas previas en el pie o pierna que comprometieran la toma de datos del presente estudio. El presente estudio se realizó respetando las recomendaciones internacionales sobre investigación de la Declaración de Helsinki de la Asociación Médica Mundial ${ }^{15}$.

La disección se llevó a cabo retirando cuidadosamente la piel y el tejido celular subcutáneo de la cara lateral y dorsal de la pierna y del pie para dejar expuesta la fascia profunda a dicho nivel. Durante la disección se localizó en nervio sural, el nervio cutáneo dorsal intermedio y el nervio cutáneo dorsal medial, evitando en todo lo posible la distorsión de las estructuras nerviosas. Para ello, el recorrido de los nervios se siguió durante la disección proximalmente hasta su punto de perforación en la fascia profunda y distalmente hasta las terminaciones digitales, respetando su trayecto sin movilizarlos y únicamente apartando la grasa alrededor del nervio para una mejor visualización del mismo.

Una vez que los nervios estaban identificados y su recorrido correctamente delimitado, se anotó el patrón de perforación y de división del nervio peroneo superficial en la pierna siguiendo la clasificación propuesta por Tomaszewski y cols. ${ }^{13}$, que establece tres patrones de división del nervio peroneo superficial: 1) Tipo I: el nervio peroneo superficial perfora la fascia profunda y posteriormente se divide en nervio cutáneo dorsal intermedio y nervio cutáneo dorsal medial (Figura 1); 2) Tipo II: el nervio peroneo superficial se divide en nervio cutáneo dorsal intermedio y nervio cutáneo dorsal medial en la cara profunda de la pierna antes de perforar la fascia profunda y posteriormente los dos nervios perforan la fascia como dos estructuras independientes (Figura 3), y 3) Tipo III: el nervio 


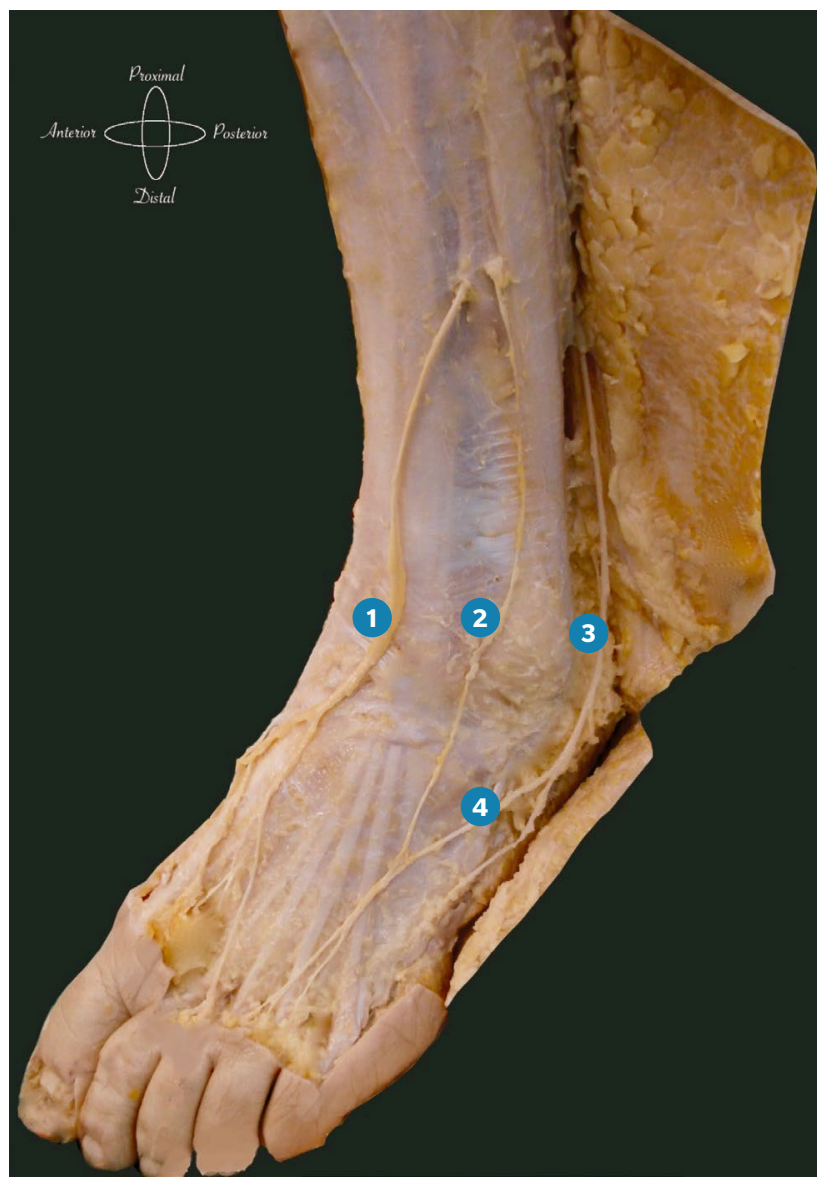

Figura 3. Patrón de inervación tipo II de la clasificación descrita por Tomaszewski y cols. ${ }^{13}$. Nótese cómo el nervio peroneo superficial se bifurca en nervio cutáneo dorsal medial e intermedio antes de atravesar la fascia profunda. Ambos nervios realizan una perforación de forma independiente por el compartimento anterior de la pierna.

1. Nervio cutáneo dorsal medial; 2. Nervio cutáneo dorsal intermedio; 3. Nervio sural; 4. Rama anastomótica del nervio sural al nervio cutáneo dorsal intermedio.

peroneo superficial perfora la fascia profunda y continúa su curso de manera similar al nervio cutáneo dorsal medial y el nervio cutáneo dorsal intermedio está ausente. En este patrón de inervación el territorio de inervación del nervio cutáneo dorsal intermedio es suplido por el nervio sural (Figura 4).

A su vez, se tomaron las siguientes mediciones en el plano sagital con el pie y la pierna desde una posición lateral, el tobillo a $90^{\circ}$ y tomando como referencia el punto más distal del maléolo peroneo: 1) distancia entre el punto más distal del maléolo peroneo al punto de perforación del nervio peroneo superficial en la fascia profunda en el patrón tipo I de Tomaszewski y cols. ${ }^{13}$, o al punto de perforación del nervio cutáneo dorsal intermedio y al nervio cutáneo dorsal medial en el patrón tipo II de Tomaszewski y cols. ${ }^{13}$; b) distancia entre el punto más distal del maléolo peroneo al punto de división del nervio peroneo superficial en nervio cutáneo dorsal intermedio y nervio cutáneo dorsal medial (únicamente en el patrón tipo I de Tomaszewski y cols. ${ }^{13}$ ); c) distancia entre el punto más distal del maléolo peroneo al nervio sural en dirección distal siguiendo el eje longitudinal de la pierna (Figura 5). Todas las mediciones y valoraciones fueron realizadas por el mismo investigador (J. P. H.). Para la recogida de datos en cada pie incluido en el estudio el patrón de distribución y las mediciones realizadas desde el maléolo lateral se dibujaron en un diagrama del pie que posteriormente se pasó a una tabla de datos. Para realizar las mediciones se utilizó una regla en milímetros.

Se realizó un análisis descriptivo de los datos obtenidos en las mediciones cadavéricas y se calcularon los porcentajes para las variables cualitativas, así como las medias y las desviaciones estándar para las variables cuantitativas. Se calculó el intervalo de confianza para el $95 \%$ de las mediciones tomadas.

\section{RESULTADOS}

Se incluyeron un total de 49 piezas anatómicas (pies) en el estudio. La lateralidad de los pies estudiados fue de 30 pies derechos (61.22 \%) y 19 de pies izquierdos (38.77\%). Los datos referentes al sexo y raza de los especímenes no pudieron ser obtenidos.

La distribución del nervio peroneo superficial encontrada fue la siguiente: 22 casos (48.89\%; IC 95\% de $33.70 \%$ a $64.08 \%$ ) de los especímenes estudiados presentaba una distribución tipo I, 15 de los casos (33.33\%; IC $95 \%$ de $19.01 \%$ a $47.66 \%$ ) de los especímenes estudiados presentaba una distribución tipo II y únicamente 8 casos (17.78\%; IC $95 \%$ de $6.16 \%$ a $29.39 \%$ ) de los especímenes estudiados presentaban una distribución tipo III. En 4 de los casos no pudo identificarse el patrón de inervación a través de la disección por errores técnicos en la misma o porque la pieza cadavérica estaba seccionada muy distalmente y no permitía la valoración de su patrón de inervación entre tipo I y tipo II en la zona proximal.

Finalmente, la Tabla I recoge los resultados de las mediciones obtenidas en los especímenes estudiados con la media y los intervalos de confianza al $95 \%$.

\section{DISCUSIÓN}

Las estructuras superficiales del dorso del pie presentan una inervación compleja y altamente variable de unos individuos a otros. Los estudios existentes sobre la inervación dorsal del pie muestran variaciones importantes en cuanto a la distribución y al territorio de inervación del nervio cutáneo dorsal intermedio y nervio sural en la cara dorsolateral del pie y pierna, aunque los porcentajes varían ampliamente de unos estudios a otros sin un claro consenso en este sentido. Varios estudios cifran la ausencia del nervio cutáneo dorsal intermedio entre un $10 \%^{7}$ y un $24-35 \% \%^{4,5,9}$, mientras que otros 
Figura 4. Patrón de inervación tipo III de la clasificación descrita por Tomaszewski y cols. ${ }^{13}$. Nótese cómo el nervio peroneo superficial sigue el trayecto del nervio cutáneo dorsal medial (flechas azules) con ausencia del nervio cutáneo dorsal intermedio.

El nervio sural (flechas amarillas) aporta la inervación correspondiente al territorio del nervio cutáneo dorsal intermedio.

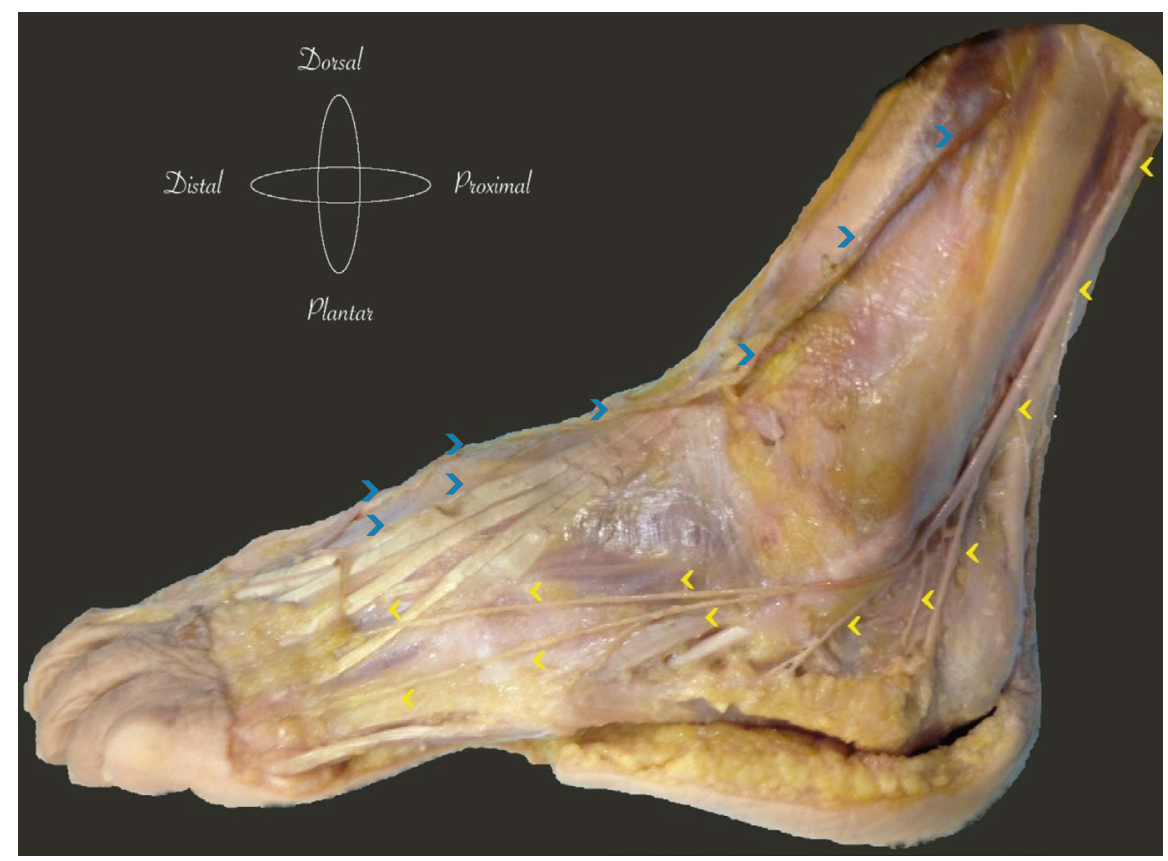

estudios lo citan en un $0 \%{ }^{10-12}$ con un metanálisis reciente de Tomazewski y cols., cifrando esta variación en un $1.8 \%$ con un IC al $95 \%$ de $0 \%$ a $4.9 \%{ }^{13}$.

El presente estudio ha mostrado un porcentaje de incidencia, tomando el patrón de inervación de Tomazewski y cols. ${ }^{13}$, de aproximadamente la mitad de casos (48.89\%; IC $95 \%$ de $33.70 \%$ a $64.08 \%$ ) de patrón tipo I en el que el nervio peroneo superficial perfora la fascia profunda y posteriormente se divide en nervio cutáneo dorsal intermedio y nervio cutáneo dorsal medial (Figura 1), un tercio de casos (33.33\%; IC $95 \%$ de $19.01 \%$ a $47.66 \%$ ) de patrón tipo II en el que el nervio peroneo superficial se divide en nervio cutáneo dorsal intermedio y nervio cutáneo dorsal medial en la cara profunda de la pierna y perforan la fascia como dos estructuras independientes (Figura 3), y un $17.78 \%$ (IC $95 \%$ de $6.16 \%$ a $29.39 \%$ ) de patrón tipo III con ausencia de nervio cutáneo dorsal intermedio y cuyo territorio de inervación es suplido por el nervio sural (Figura 4). Estos resultados son bastante similares a los reportados en otros estudios en cuanto a la presencia del patrón tipo I $\mathrm{I}^{4,10,11}$ que la mayoría de estudios estiman en una frecuencia ligeramente superior al $50 \%$. Los resultados del presente estudio apoyan estos datos. En este contexto es importante señalar que, a pesar de que la mayoría de los textos anatómicos clásicos describen únicamente este patrón para describir la inervación del dorso del pie ${ }^{1-3}$, este patrón ocurre únicamente en la mitad de los especímenes estudiados, siendo bastante común encontrar variaciones anatómicas a esta descripción.

El nervio sural es clínicamente importante porque es frecuentemente utilizado para realizar test conducción nerviosa, para biopsia en enfermedades neuromusculares como la neuropatía hereditaria sensitivo-motora e incluso utilizado como

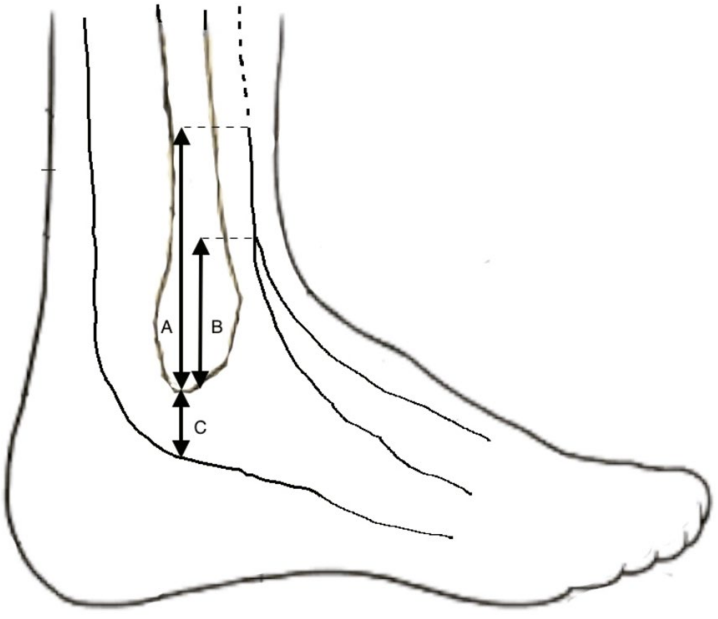

Figura 5. Diagrama de las mediciones realizadas en los especímenes del estudio. A: distancia en el eje de la pierna desde el punto más distal del maléolo peroneo al punto de perforación del nervio peroneo superficial en la fascia profunda. B: distancia en el eje de la pierna desde el punto más distal del maléolo peroneo al punto de bifurcación del nervio peroneo superficial en nervio cutáneo dorsal medial y cutáneo dorsal intermedio. C: distancia en el eje vertical de la pierna desde el punto más distal del maléolo peroneo al nervio sural.

injerto de nervio en cirugía plástica y reparadora. Este nervio presenta una gran variedad en su formación y su distribución en el dorso del pie. Es la opinión de los autores del presente manuscrito que el papel del nervio sural en el dorso del pie es mucho más amplio al descrito en la literatura clásica como únicamente inervando la cara lateral del quinto dedo. El presente estudio ha mostrado que en un $17.78 \%$ (IC $95 \%$ de 
Tabla I. Tabla descriptiva de las mediciones obtenidas en el estudio

\begin{tabular}{|c|c|c|c|c|c|}
\hline & \multirow[b]{2}{*}{ n. ${ }^{\circ}$ casos } & \multirow[b]{2}{*}{ Media $(\mathrm{cm})$} & \multirow[b]{2}{*}{$\mathrm{DS}(\mathrm{cm})$} & \multicolumn{2}{|c|}{ IC (95\%) } \\
\hline & & & & $\begin{array}{c}\text { Límite } \\
\text { Inferior }(\mathrm{cm})\end{array}$ & $\begin{array}{l}\text { Límite } \\
\text { Superior }(\mathrm{cm})\end{array}$ \\
\hline $\begin{array}{l}\text { Distancia del maléolo peroneo al punto de perforación } \\
\text { nervio peroneo superficial (Tipo I) }\end{array}$ & 32 & 9.81 & 4.40 & 8.22 & 11.40 \\
\hline $\begin{array}{l}\text { Distancia del maléolo peroneo al punto de división del } \\
\text { nervio peroneo superficial (Tipo I) }\end{array}$ & 22 & 5.05 & 2.67 & 3.86 & 6.23 \\
\hline $\begin{array}{l}\text { Distancia del maléolo peroneo al punto de perforación } \\
\text { nervio cutáneo dorsal medial (Tipo II) }\end{array}$ & 13 & 7.20 & 6.05 & 3.54 & 10.85 \\
\hline $\begin{array}{l}\text { Distancia del maléolo peroneo al punto de perforación } \\
\text { nervio cutáneo dorsal intermedio (Tipo II) }\end{array}$ & 13 & 7.53 & 3.22 & 5.58 & 9.47 \\
\hline $\begin{array}{l}\text { Distancia del maléolo peroneo al punto más distal del } \\
\text { nervio sural }\end{array}$ & 46 & 1.94 & 0.41 & 1.82 & 2.06 \\
\hline
\end{tabular}

cm: centímetros. DS: desviación estándar. IC (95\%): intervalo de confianza al $95 \%$.

$6.16 \%$ a $29.39 \%$ ) de los casos hay una ausencia del nervio cutáneo dorsal intermedio y su territorio es suplido por el nervio sural (Figura 4). No obstante, los resultados del presente estudio son algo menores respecto a la presencia del patrón tipo III, que los descritos por Thompson y cols. ${ }^{4}$ con un $24 \%$, Solomon y cols. ${ }^{5}$ con un $35 \%$ y Aktan-Ikiz y Urcerler ${ }^{9}$ con un $26.7 \%$, aunque apoyan la idea de que esta variación anatómica podría estar presente en un porcentaje significativo de la población y no suponer una variación marginal como supone el metanálisis de Tomazewski y cols. datando su frecuencia en un $1.8 \%^{13}$. Además de esto, y aunque el presente estudio no midió este aspecto, es común la presencia de una anostomosis entre el nervio sural y nervio cutáneo dorsal intermedio (Figura 1 y Figura 2) en más de la mitad de la población ${ }^{16}$. Jeong y cols. ${ }^{17}$ estudiaron el origen y recorrido del nervio sural en 110 casos y encontraron al nervio sural dando inervación al $5 .^{\circ}, 4^{\circ}$ dedo y cara medial del $3^{\text {er }}$ dedo en un $53.2 \%$ de casos, y al $5 .{ }^{\circ}$ y $4 .{ }^{\circ}$ dedo en un $33.3 \%$ de casos. Todos estos datos hacen pensar que el nervio sural tiene un papel más relevante en la inervación del dorso del pie que el clásicamente descrito y no se limita únicamente a la cara lateral del quinto dedo.

Por su parte, las mediciones obtenidas en el presente estudio del patrón de perforación del nervio cutáneo dorsal intermedio en el patrón tipo I (48.89\%) han mostrado que el IC al $95 \%$ se encuentra entre $8.22 \mathrm{~cm}$ y $11.40 \mathrm{~cm}$ del punto más distal del maléolo externo. Estos datos podrían ser comparables a los obtenidos en otros estudios que midieron el mismo parámetro con los mismos puntos de referencia. Blair \& Botte ${ }^{10}$ citan la distancia del punto de perforación del nervio peroneo superficial con el tobillo (no con el punto distal del maléolo externo) en $12.3 \mathrm{~cm}$; Cánovas y cols. ${ }^{8}$ reportan la distancia con respecto al maléolo externo en $11 \mathrm{~cm}$, Sayli y cols. en $10.41 \mathrm{~cm}^{11}$ y Solomon y cols. en $9.1 \mathrm{~cm}^{5}$. Los resultados del presente estudio concuerdan con dichos datos y parece que el intervalo de confianza creado (entre $8.22 \mathrm{~cm}$ y $11.40 \mathrm{~cm}$ desde la punta del maléolo externo) podría ser un buen punto de referencia para encontrar la perforación del nervio peroneo superficial en la fascia cruris y podría ser un punto para usarse como referencia en los bloqueos anestésicos del pie. No obstante, es interesante recordar que, según nuestro estudio, este patrón ocurre únicamente en el tipo I y el tipo III y no en el tipo II en el que los nervios cutáneo dorsal intermedio y cutáneo dorsal medial perforan la fascia de forma independiente.

En este último supuesto (patrón tipo II), la distancia calculada desde el maléolo externo a las perforaciones al nervio cutáneo dorsal intermedio y medial es mucho más variable, como indican las desviaciones estándar y los intervalos de confianza calculados. El motivo de esta variabilidad posiblemente sea debido al compartimento en el que se encuentran ambos nervios en el momento de la perforación. Se ha descrito que la perforación del nervio cutáneo dorsal intermedio puede realizarse desde el compartimento anterior de la pierna o desde el compartimento lateral tanto desde una posición anterior al peroné o incluso desde una posición posterior a este ${ }^{10,11,18}$, aspecto que no se analizó en el presente estudio. Las distintas posiciones desde las que se produce la perforación de ambos nervios hacen que el punto de perforación sea más variable y más difícil de reproducir en el patrón tipo II. No obstante, en el presente estudio la perforación de la fascia cruris del nervio cutáneo dorsal intermedio es más proximal que la del nervio cutáneo dorsal medial en el patrón tipo II (Figura 2), aunque la diferencia de ambas perforaciones con respecto al maléolo peroneo es muy pequeña.

Respecto a la medición realizada del nervio sural, el presente estudio calculó la distancia desde la punta distal del maléolo externo al nervio sural al punto más distal del nervio sural siguiendo el eje longitudinal del peroné. Esta distancia obtuvo un valor de $1.94 \mathrm{~cm}$ (IC $95 \%$ de $1.82 \mathrm{~cm}$ a $2.06 \mathrm{~cm}$ ). Un metanálisis publicado por Ramakrishnan y cols. en 2015 sobre las variaciones anatómicas en la formación y curso del nervio sural ${ }^{19}$, utilizaron 6 estudios (185 miembros inferiores) en los que se había calculado la distancia vertical existente la punta distal del maléolo peroneo al nervio sural para calcular 
esta distancia encontrando una distancia media de $1.53 \mathrm{~cm}$ $(S D=0.56)$. Aunque los datos del presente estudio distan levemente de los calculados por Ramakrishnan y cols., parece que entre $1.5 \mathrm{~cm}$ y $2 \mathrm{~cm}$ distal al maléolo peroneo sería una buena localización para el nervio sural, lo que podría utilizarse como un punto de referencia para el bloqueo anestésico del pie. No obstante, serían necesarios estudios clínicos con bloqueos anestésicos en dicha localización para comprobar esta hipótesis.

Existen limitaciones asociadas al estudio, por lo que los resultados deben ser analizados con cautela. En primer lugar, es posible que la raza y el sexo de los especímenes del estudio puedan condicionar los patrones de distribución de la inervación dorsal del pie con un menor porcentaje de patrón tipo I en estudios europeos comparado con estudios en $\mathrm{Asia}^{13}$. En el presente estudio los datos de raza y sexo de los especímenes estudiados no pudieron ser obtenidos, por lo que no sabemos si esto ha podido influenciar en los resultados finales obtenidos y la comparación con otros estudios podría ser más compleja. En segundo lugar, las mediciones realizadas se llevaron a cabo de forma manual con una regla en milímetros por un mismo investigador sin utilizar ningún instrumento calibrado o testado en su fiabilidad, lo que podría añadir sesgos o inexactitudes a las mediciones del estudio. Finalmente, las mediciones del presente estudio fueron realizadas en milímetros sin ninguna normalización al tamaño del pie. Cabría preguntarse si la medición en porcentajes de medición como el utilizado en el estudio de Patel y cols. ${ }^{13}$, en los que toman como referencia segmentos anatómicos concretos como la longitud del peroné, podría haber sido más útil desde el punto de vista técnico (por ejemplo los $9.81 \mathrm{~cm}$ calculados de media entre el maléolo peroneo y el punto de perforación del nervio peroneo superficial podrían ser muy diferentes entre un individuo de $190 \mathrm{~cm}$ de estatura y otro de $150 \mathrm{~cm}$ ), lo cual puede poner en duda la utilidad de estas mediciones en determinados individuos.

En conclusión, existe controversia en la literatura con respecto a la inervación dorsal del pie, sus variantes anatómicas y sus frecuencias y el presente estudio aporta más datos y bibliografía en este sentido. En la presente serie estudiada los datos encontrados fueron de un $48.89 \%$ (IC $95 \%$ de $33.70 \%$ a $64.08 \%$ ) de casos con una distribución tipo I (nervio peroneo superficial perfora la fascia profunda y posteriormente se divide en nervio cutáneo dorsal intermedio y nervio cutáneo dorsal medial), un $33.33 \%$ (IC $95 \%$ de $19.01 \%$ a $47.66 \%$ ) una distribución tipo II (nervio peroneo superficial se divide en nervio cutáneo dorsal intermedio y nervio cutáneo dorsal medial en la cara profunda de la pierna antes de perforar la fascia profunda y posteriormente los dos nervios perforan la fascia como dos estructuras independientes) y un $17.78 \%$ (IC $95 \%$ de $6.16 \%$ a $29.39 \%$ ) con una distribución tipo III (nervio peroneo superficial perfora la fascia profunda y continúa su curso de manera similar al nervio cutáneo dorsal medial y el nervio cutáneo dorsal intermedio está ausente).

\section{CONFLICTO DE INTERESES}

Los autores no presentan conflictos de intereses relevantes con el presente artículo.

\section{FINANCIACIÓN}

Los autores declaran no haber tenido ninguna financiación pública ni privada para la realización del presente estudio.

\section{BIBLIOGRAFÍA}

1. Sarrafian SK, Kellikian AS. Nerves. En: KellikianAS, ed. Sarrafian's anatomy of the foot and ankle: descriptivie, topografphical, functional. $3^{a} \mathrm{ed}$. Philadelphia: Walters Kluwer Lippincott Williams \& Wilkins; 2011. p. 381-427.

2. Drake R, Vogl AW, Mitchell AWM, eds. Gray's anatomy for students. $3^{\text {rd }}$ Ed. Philadelphia: Elsevier; 2015.

3. Moore KL, Dalley AF, Agur AM, eds. Clinically Oriented Anatomy. $7^{\text {th }}$ Ed. Philadelphia: Lippincott Williams \& Wilkins; 2014.

4. Thompson A. Second Annual Report of the Committee of Collective Investigation of the Anatomical Society of Great Britain and Ireland for the year 1890-1891. J Anat Physiol. 1891;26(Pt 1):76-90.

5. Solomon LB, Ferris L, Tedman R, Henneberg M. Surgical anatomy of the sural and superficial fibular nerves with an emphasis on the approach to the lateral malleolus. J Anat. 2001;199(Pt 6):717-23.

6. Kosinski $C$. The course, mutual relations and distribution of the cutaneous nerves of the metazonal region of the leg and foot. J Anat and Physiol. 1926;60(Pt 3):274-97

7. Ucerler $\mathrm{H}, \mathrm{Ikiz} \mathrm{A}$. The variations of the sensory branches of the superficial peroneal nerve course and its clinical importance. Foot Ankle Int. 2005:26(11):942-6. DOI: 10.1177/107110070502601108.

8. Canovas F, Bonnel F, Kouloumdjian P. The superficial peroneal nerve at the foot. Organization, surgical applications. Surg Radio Anat. 1996;18(3):241-4

9. Aktan IkizZA, Ucerler $\mathrm{H}$. The distribution of the superficial peroneal nerve on the dorsum the foot and its clinical importance in flap surgery. Foot Ankle Int. 2006;27(6):438-44. DOI: 10.1177/107110070602700609.

10. Blair LM, Botte MJ. Surgical anatomy of the superficial peroneal nerve in the ankle and foot. Clin Orthop Relat Res. 1994;305:229-38.

11. Sayli U, Tekdemyr Y, Cubuk HE, et al. The course of the superficial peroneal nerve: an anatomical cadaver study. Foot Ankle Int. 1998;4:63-9.

12. Pacha A, Carrera A, Llusa M, Permanyer E, Molona O, Morro R. Clinical anatomy of the superficial peroneal nerve in the distal leg. Eur J Anat. 2003;7(Suppl. 1):15-20.

13. Tomaszewski KA, Graves MJ, Vikse J, Pękala PA, Sanna B, Henry BM, et al. Superficial fibular nerve variations of fascial piercing: A meta-analysis and clinical consideration. Clin Anat. 2017;30(1):120-5. DOI: 10.1002/ ca. 22741 .

14. Tomaszewski KA, Henry BM, Ramakrishnan PK, Roy J, Vikse J, Loukas $M$, et al. Development of the Anatomical Quality Assurance (AQUA) Checklist: guidelines for reporting original anatomical studies. Clin Anat. 2017;30(1):14-20. DOI: 10.1002/ca.22800.

15. World Medical, Assembly. Declaration of Helsinki. Ginebra, Suiza: World Health Organization; 1964.

16. Drizenko A, Demondion X, Luyckx F, Mestdagh H, Cassagnaud X. The communicating branches between the sural and superficial peroneal nerves in the foot: a review of 55 cases. Ser Radiol Anat. 2004;26(6):44752. DOI: 10.1007/s00276-004-0264-9.

17. Jeong SK, Paik DJ, Hwang YI. Variations in Sural nerve Fomation Pattern and distribution on the dorsum or the foot. Clin Anat. 2017;30(4):52532. DOI: 10.1002/ca.22873.

18. Adkinson DP, Bossem J, Gaccione DR, Gabriel KR. Anatomical variations in the course of the superficial peroneal nerve. J Bone Joint Surg Am. 1991;73(1):112.

19. Ramakrishnan PK, Henry BM, Vikse J, Roy J, Saganiak K, Mizia E, et al. Anatomical variations of the formation and course of the sural nerve: $A$ systematic review and meta-analysis. Ann Anat. 2015;202:36-44. DOI: 10.1016/j.aanat.2015.08.002 